\title{
Spinal Cord - Skeletal Muscle Cocultures Detect Muscle-Relaxant Action of Botulinum Neurotoxin A
}

\author{
Veit-Simon Eckle ${ }^{1, *}$, Berthold Drexler ${ }^{1, *}$, Christian Grasshoff ${ }^{1}$, Thomas Seeger ${ }^{2}$, \\ Horst Thiermann ${ }^{2}$ and Bernd Antkowiak ${ }^{1}$ \\ ${ }^{1}$ Department of Anesthesiology and Intensive Care Medicine, Experimental Anesthesiology Section, Eberhard-Karls University \\ Tübingen, Germany; ${ }^{2}$ Bundeswehr Institute of Pharmacology and Toxicology, Munich, Germany
}

\begin{abstract}
Summary
The mouse $L D_{50}$ assay is routinely used for potency testing of botulinum toxins. Unfortunately, this test is associated with severe pain and distress in animals and consumes large numbers of mice. Here we used cocultures of spinal cord and muscle tissue to assess botulinum toxin potency. Cocultures were prepared from mouse embryonic tissue (C57/BL6J) and cultured for 24-27 days. Spontaneous muscle activity was quantified in sham-and botulinum toxin-treated cultures for up to 3 days by video microscopy. At a concentration of $58 \mathrm{fmol} / \mathrm{l}$ or higher, incobotulinumtoxin A significantly reduced the frequency of muscle contractions within $24 \mathrm{~h}$ after exposure. Hence, the sensitivity of nerve-muscle cultures is comparable to that of the mouse $L D_{50}$ assay. The limit of detection observed in this study is close to that of the most sensitive cell-based bioassays, capable of detecting concentrations of botulinum neurotoxin A of between 30 and $50 \mathrm{fmol} / \mathrm{l}$. However, spontaneous muscle activity of individual cultures displayed considerable fluctuations when evaluated on a day-to-day basis and, in its present form, this in vitro assay might be too laborious for botulinum toxin potency testing. Thus, methodical improvements to decrease data variability and increase throughput are the next milestones towards developing the model into an assay that can be utilized to reduce animal experimentation.
\end{abstract}

Keywords: organotypic culture, botulinum toxin $A$, incobotulinumtoxin $A, L D_{50}$ assay, nerve-muscle culture

\section{Introduction}

Botulinum neurotoxins are zinc endopeptidases which hydrolyze synaptosomal-associated receptor (SNARE) proteins (Martens and McMahon, 2008). By cleaving the plasma membrane-associated peptide SNAP-25, botulinum toxin A inhibits the release of acetylcholine at the neuromuscular junction and causes flaccid paralysis that can be fatal (Humeau et al., 2000).

Medicinal products containing botulinum toxin A are in current use for treating several diseases including dystonia, migraine, hyperhydrosis, blepharospasm and torticollis (Dressler, 2012; Schiavo et al., 2000). According to legal regulations, each botulinum toxin batch must undergo potency testing (Adler et al., 2010). The standard procedure according to Pharm EU and USP is the classical mouse $\mathrm{LD}_{50}$ (lethal dose that kills $50 \%$ of the tested animals) assay. Because this test is associated with severe distress in animals and requires large numbers of rodents, it is highly desirable to develop animal-free assays (Adler et al., 2010; Bitz, 2010; Pharm EU, 2011). In this respect, signifi- cant progress has been made in the past years (Fernández-Salas et al., 2012; Grate et al., 2010). However, due to patent issues it is unlikely that new assays developed by manufacturers of licensed products or profit-oriented institutions will widely replace animal $\mathrm{LD}_{50}$ tests in the near future (Fernández-Salas et al., 2012). For this reason, the promotion of in vitro assays that are free to use should not be neglected.

Recently, a cell culture model composed of spinal cord tissue slices and muscle cells (nerve-muscle-cultures) was successfully utilized for evaluating muscle-relaxant properties of succinylcholine and organophosphorus compounds (Drexler et al., 2011, 2013). In this preparation, motor neurons synapse with muscle cells and action potential activity of spinal motor neurons directly translates into muscle contractions (Braschler et al., 1989). We consider nerve-muscle cultures a valuable tool for testing botulinum toxins as, unlike other cell-culture based test systems, this model is capable of measuring the patient-relevant action of the toxins, namely muscle relaxation. In the past years, several approaches to establish these cultures have been successfully developed and published (Braschler et al., 1989;

Received April 29, 2013; accepted in revised form August 5, 2014; Epub August 14, 2014; http://dx.doi.org/10.14573/altex.1304291

*Authors contributed equally to this work. 
Gähwiler, 1981; Rosato-Siri et al., 2004; Spenger et al., 1991; Stoppini et al., 1991). Thus, free usability of these methods is warranted.

However, investigations on the actions of botulinum toxins in nerve-muscle cultures have not been carried out so far. The primary objective of the present study was to find out whether botulinum neurotoxin A concentrations corresponding to approximately $1,2,5$, and 50 mouse $\mathrm{LD}_{50}$ units diminish muscle activity in these cultures. We observed that at a concentration corresponding to 2 mouse LD50 units or higher, incobotulinumtoxin $\mathrm{A}\left(\mathrm{Xeomin}^{\circledR}\right)$ did significantly reduce muscle activity.

\section{Experimental procedures}

\section{Preparation of co-cultures}

All procedures were approved by the local animal care committee (Eberhard-Karls-University, Tubingen, Germany) and were in accordance with the German law on animal experimentation. Every endeavor was made to minimize both suffering and number of animals sacrificed for this study. Under deep anesthesia pregnant C57/BL6J mice (E 13-15) were sacrificed and embryonic tissue slices $(300 \mu \mathrm{M})$ from the spinal cord and surrounding muscles were obtained as previously reported (Braschler et al., 1989). These slices were placed on a glass coverslip and embedded into a fluid consisting of chicken plasma and thrombin (Sigma, Taufkirchen, Germany). Coagulation of the fluid occurred within a few minutes and the tissue was mechanically fixed onto the coverslip. Next, the coverslip and the attached tissue were transferred into a plastic tube and supplemented with $0.75 \mathrm{ml}$ nutrition medium composed of 25 vol-\% horse serum (Invitrogen, Karlsruhe, Germany), 25 vol-\% Hank's balanced salt solution (Sigma) and 50 vol-\% Basal Medium Eagle (Sigma) with neuronal growth factor (Sigma) added to a final concentration of $10 \mathrm{nM}$. The culture medium and the tissue slices therein were incubated with $5 \% \mathrm{CO}_{2}$ at $36^{\circ} \mathrm{C}$ for $2-3$ hours. Thereafter, the plastic tubes were tightly sealed by screw caps. After one day in culture, antimitotics (10 $\mu \mathrm{M}$ 5-fluoro-2-deoxyuridine, $10 \mu \mathrm{M}$ cytosine-b-arabinofuranoside, $10 \mu \mathrm{M}$ uridine; all from Sigma) were added in order to reduce the growth of glial cells. The roller tube approach was used for culturing the tissue (Gähwiler, 1981). We typically obtained 50-80 nerve-muscle cultures from each preparation. Spontaneous muscle contractions developed within the first week ex vivo, indicating de novo formation of neuromuscular junctions. This was observed in about $65 \%$ of the cultures. Recordings of muscle contractions were carried out in cultures after 23 days in vitro when major developmental steps in maturation had been passed (Avossa et al., 2003; RosatoSiri et al., 2004).

\section{Setup for monitoring muscle movements}

In nerve-muscle cultures, muscle contractions are controlled by acetylcholine-releasing spinal neurons that synapse with muscle fibers (Magloire and Streit, 2009; Rosato-Siri et al., 2004). Botulinum toxin A is known to inhibit acetylcholine release at the neuromuscular junction by cleaving SNAP-25
(Humeau et al., 2000; Schiavo et al., 2000; Turton et al., 2002). We therefore expected a reduction of muscle activity after exposing the cocultures to incobotulinumtoxin A. Muscle movements were monitored before and during drug exposure by a video camera (The Imaging Source Europe $\mathrm{GmbH}$, Bremen, Germany) mounted on an inverted microscope (Carl Zeiss Microscopy GmbH, Göttingen, Germany). The duration of a single recording was 180 seconds and the frame rate was $30 \mathrm{~Hz}$. For performing videomicroscopic recordings, a plastic tube containing a nerve-muscle culture was placed on the heated stage of the inverted microscope. Throughout all recordings, the temperature was kept close to $36^{\circ} \mathrm{C}$. At an optic magnification of 40x, spontaneous contractions of muscle fibers were visualized. At the beginning of each recording session, contrast and brightness of the video image were optimized and a single picture was taken and stored on the computer. This allowed re-identification of the regions of interest, which was necessary as cultures were returned to the incubator between recording sessions. A small fraction of muscle fibers displayed tonic contractions. These samples were excluded from further analysis.

\section{Quantification of muscle activity}

Muscle activity was quantified offline using a Hewlett Packard Z800 video-workstation (Picturetools, Hamburg, Germany). Videos were played back and the regions of interest were defined by visual inspection. Close attention was paid that these regions contained high-contrast borders of muscle fibers that were moving in the course of muscle contractions. Due to the muscle movements, the brightness of pixels ( 8 bit resolution) in the region of interest changed with time. These changes were well correlated with the intensity and frequency of muscle contractions (Drexler et al., 2011). An in-house developed algorithm was used for quantifying the changes in brightness between subsequent video frames (Drexler et al., 2011, 2013). Software for analyzing the data was written in Matlab 7.1 (The MatWorks Inc., Natick, MA, USA). The correctness and performance of the algorithms were confirmed by self-designed test videos. The latter were created in Adobe After Effects CS4 (Adobe Systems, Inc., San Jose, CA, USA).

\section{Pre-treatment of co-cultures}

In nerve-muscle cultures, synapses between motor neurons and muscle cells and, in addition, synapses between spinal interneurons of the ventral horn area are accessible to drugs dissolved in the culture medium. Botulinum toxins do not exclusively act at neuromuscular junctions but also inhibit transmitter release at neuronal synapses (Antonucci et al., 2008; Capogna et al., 1997; Scarlatos et al., 2008). In spinal networks, glutamate is the major excitatory neurotransmitter. Thus, it seemed possible that in our study incobotulinumtoxin A decreased muscle activity at least in part via a spinal mechanism of action, namely by reducing glutamate release onto spinal motor neurons. To exclude this possible pathway of action, cultures were treated with glutamate receptor antagonists (D-L-2-amino-5-phosphonopentanoic acid (AP5; $20 \mu \mathrm{M}$ ) and 6-cyano-7-nitroquinoxaline-2.3-dione (CNQX; $15 \mu \mathrm{M})$ ) prior to exposing them to in- 
cobotulinumtoxin A. This pre-treatment only mildly affected spontaneous muscle activity, which is consistent with findings of a previous study (Magloire and Streit, 2009).

\section{Chemicals}

Unexpired batches of Xeomin ${ }^{\circledR}$ (batch \# 050425, Merz Pharmaceuticals, Frankfurt, Germany) were used in the present study. Vials contained 100 mouse LD50 units of botulinum toxin type A (corresponding to $0.44 \mathrm{ng}$ total clostridial protein content), $1.0 \mathrm{mg}$ human serum albumin, and $4.7 \mathrm{mg}$ sodium sucrose (Dressler, 2012). Lyophilized botulinum toxin A complex $(0.44 \mathrm{ng})$ was diluted with culture medium containing the glutamate-receptor antagonists AP5 $(20 \mu \mathrm{M})$ and CNQX $(15 \mu \mathrm{M})$. The toxin was added to a final concentration corresponding to 1, 2, 5 and 50 mouse LD50 units, respectively. Assuming a molecular weight of $150 \mathrm{kDa}$, the final concentration of botulinum toxin A corresponding to one LD50 unit was $29 \mathrm{fmol} / 1$.

\section{Experimental design}

Spontaneous muscle activity in nerve-muscle cultures was recorded over a period of four subsequent days. Prior to the present study, it was unknown whether muscle activity spontaneously changes within this period of observation. In that case, pharmacological interventions would not be feasible. To exclude this possibility, two experimental groups were defined. The first group was treated with the neurotoxin. Cultures of the second group (sham) underwent all manipulations that were performed

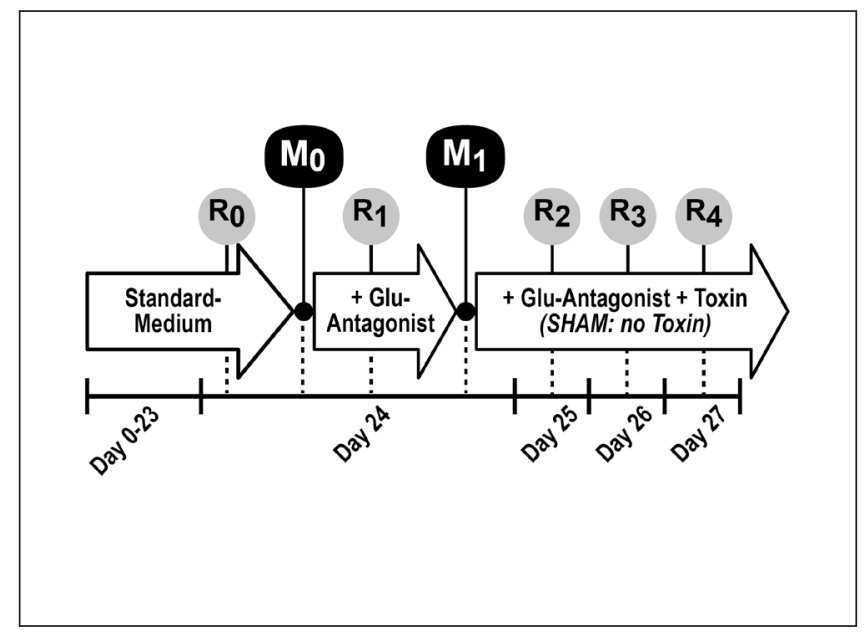

Fig. 1: Experimental workflow used for quantifying the actions of botulinum toxin $A$ in nerve-muscle cultures Cell cultures derived from the same preparation were divided into a botulinum toxin group and a sham group. After 23 days ex vivo, muscle activity was recorded in the cultures bathed in standard cell culture medium (R0). Then, the medium was exchanged (M0) and antagonists of glutamate receptors (+glu-antagonist) were added to both the botulinum toxin and sham group. Another recording was performed after $2 \mathrm{~h}$ incubation time (R1). Next, the cell culture medium was exchanged again (M1) and the cultures in the toxin group were exposed to incobotulinumtoxin $A$ (Xeomin $\left.{ }^{\circledR}\right)$. Then muscle activity was recorded after 24,48 and 72 h (R2, R3, R4) in both groups. in the botulinum toxin group except for the application of incobotulinumtoxin A to the culture medium. Furthermore, care was taken that cultures of the botulinum toxin and sham group were recruited from the same preparations and therefore were of the same age. Using this approach, it was possible to isolate the specific action of incobotulinumtoxin A and to quantify spontaneous baseline changes in muscle activity not associated with the action of incobotulinumtoxin A. The distribution of the data was tested by the Lilliefors test. The Kruskal-Wallis test combined with a Dunn's multiple comparison analysis was applied for statistical testing of unpaired samples assuming a non-parametric data set. Data is presented as median and interquartile range. The whiskers of the box plots signify the 2.5 to 97.5 percentile. Significant p-values are indicated with asterisks $(*<0.05, * *<0.01, * * *<0.001)$.

\section{Workflow}

Figure 1 displays the time schedule used for the pharmacological manipulations and for monitoring muscle contractions. The nerve-muscle cultures were fabricated on day 0 . Thereafter, they were maintained for at least 23 days ex vivo. During this period, functional synaptic contacts formed, innervating cocultured muscle tissue (Spenger et al., 1991; Streit et al., 1991). Muscle activity was recorded before and after adding the glutamate-receptor blockers and then subsequently at intervals of 24 hours.

\section{Results}

\subsection{Basal activity in nerve-muscle cultures}

Figure 2 shows the typical microscopic view of a nerve-muscle culture. A $300 \mu \mathrm{M}$ thick transverse spinal cord slice was cultured together with the dorsal root ganglia and muscle tissue for 24 days. Recordings were carried out in a total of 255 nerve-muscle cultures displaying robust muscle activity. In these cultures, muscle fibers contracted spontaneously. The frequency of contractions was well approximated by a normal distribution (Fig. 3, mean: $1.56 \mathrm{~Hz}, \mathrm{SD}$ : $0.92 \mathrm{~Hz}$ ). Muscle movements were abolished by the muscle-relaxant drug succinylcholine, which targets nicotinic acetylcholine receptors, thereby interrupting neuromuscular transmission (Drexler et al., 2013). These findings confirmed that muscle contractions in nerve-muscle cultures were triggered by spinal motor neurons generating action potentials and releasing acetylcholine onto muscle fibers.

Botulinum toxin A does not only reduce transmitter release at neuromuscular junctions, but also at synapses between spinal interneurons (Bigalke et al., 1985; Pellett et al., 2007; Sheridan et al., 2005; Welch et al., 2000). Glutamate is the major excitatory neurotransmitter in the spinal cord (Talpalar and Kiehn, 2010). Since motor neurons express glutamate receptors, we wondered whether a decrease in glutamate release caused by botulinum toxins might influence muscle activity. As a selective application of the toxin to spinal neurons and muscle fibers was technically impossible, we simulated the toxin's potential depression of glutamatergic synaptic transmission by applying glutamate receptor antagonists. Pharmacologic blockade of 


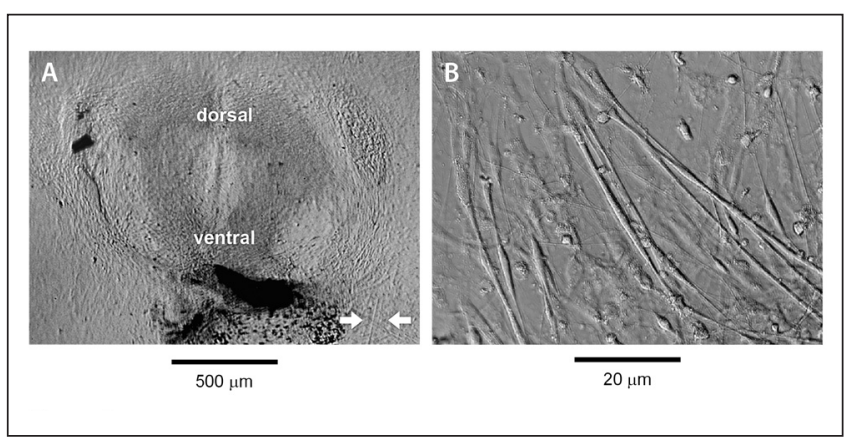

Fig. 2: Microscopic view of a typical nerve-muscle culture maintained for 24 days in vitro

(A) A spinal cord slice was cultured together with adjacent muscle tissue. A clearly visible set of muscle fibers is marked by the white arrows. (B) Muscle fibers at a higher magnification.

glutamate receptors by AP-5 $(20 \mu \mathrm{M})$ and CNQX $(15 \mu \mathrm{M})$ did not significantly reduce the frequency of muscle contractions of single muscle fibers (data not shown). Therefore, it seems unlikely that incobotulinumtoxin A was capable of attenuating the frequency of muscle contractions via this route. However, there is ample evidence in the literature that a depression of synaptic transmission produced by pharmacologic interventions evokes homeostatic responses and changes neuronal excitability on a slow time scale (Davis, 2006; Galante et al., 2000; Turrigiano, 1999). Thus, a modulating botulinum effect at the neuromuscular junction might be veiled by alterations of glutamate transmitter release in the spinal ventral horn, where locomotor activity is triggered. In order to reduce the capability of our cultures to produce long-term homeostatic responses during botulinum toxin treatment, all cultures were pre-treated with glutamate receptor blockers at point M0 of our experimental workflow (Fig. 1).

\subsection{Incobotulinumtoxin A reduced spontaneous muscle contractions}

Spontaneous muscle activity commonly emerges during the first week ex vivo. However, at this time spinal neurons and muscle cells have not yet passed major developmental maturation steps (Avossa et al., 2003; Rosato-Siri et al., 2004). Therefore, experiments testing incobotulinumtoxin A were performed after 23 days in vitro. The effects of $1.46 \mathrm{pmol} / 1$ (50 LD50 mouse units) incobotulinumtoxin $\mathrm{A}\left(\mathrm{Xeomin}^{\circledR}\right)$ on the frequency of spontaneous muscle contractions in cultures pretreated with AP5/CNQX are presented in Figure 3. Remarkably, $24 \mathrm{~h}$ after application of incobotulinumtoxin A, muscle activity was almost abolished (Fig. 3B-C). When comparing the histograms before and after incobotulinumtoxin A treatment, the distributions of the detected muscle activity appeared to be qualitatively different (Fig. 3A-B). As the distribution was almost parametric before incobotulinumtoxin A application, a strong leftward tendency was induced by the drug. The hypothesis that the muscle activity of the botulinum-treated group describes a Gaussian distribution had to be rejected (Lilliefors test). In a cumulative distribution plot the median activity corresponds to the frequency of muscle contractions with a cumulative fraction of 0.5 (shown in Fig. 3C). This number was $1.40 \mathrm{~Hz}$ in the absence of incobotulinumtoxin A, but only $0.14 \mathrm{~Hz}$ after $24 \mathrm{~h}$ of drug exposure.

\subsection{Comparison between botulinum toxin and sham-treated cultures}

In Figure 4 the frequency of muscle contractions is compared between different botulinum toxin concentrations (corresponding to 1, 2, 5 and 50 LD50 mouse units) and sham group. As muscle activity measured in the botulinum toxin group was not normally distributed, a non-parametric method was used for statistical comparison. After pre-treating cell cultures with glutamate receptor antagonists, the frequency of muscle contractions

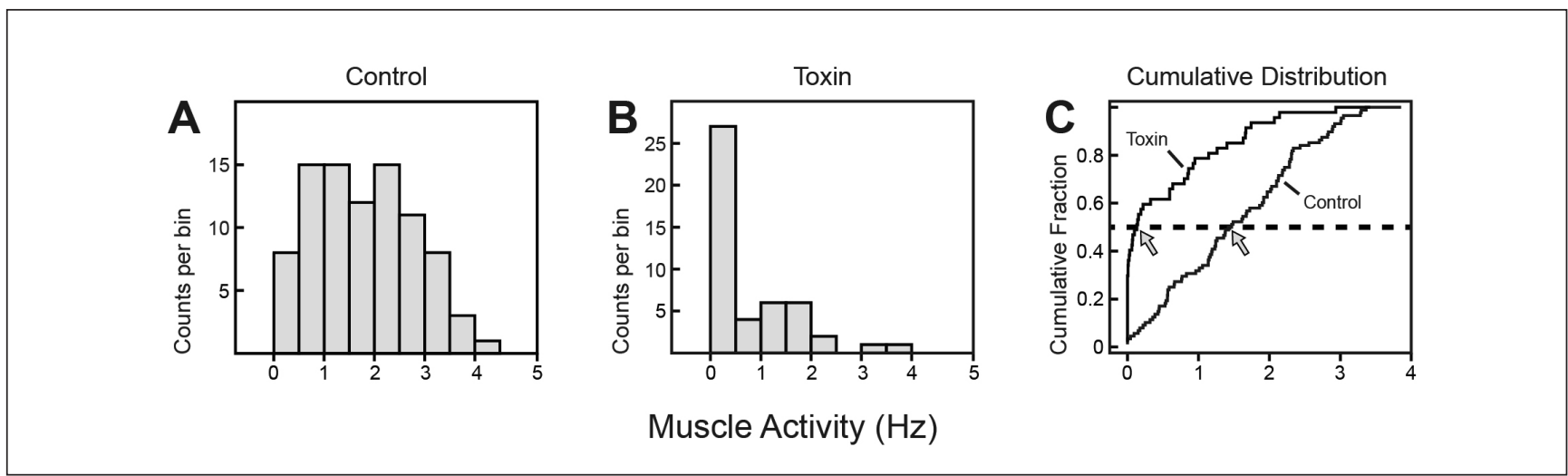

Fig. 3: The frequency of muscle contractions before and after exposing nerve-muscle-cultures to botulinum toxin $A$ (A) Before treating the cultures (control, $n=88$ ), the frequency of muscle contractions ranged between 0 and $4 \mathrm{~Hz}$ without displaying a clear peak in the bins between 0.5 and $2.5 \mathrm{~Hz}$. The frequency followed a Gaussian distribution. (B) After exposing the cultures to incobotulinumtoxin A (toxin, incubation time: $24 \mathrm{~h}, \mathrm{n}=44$ ), the distribution of muscle activity changed dramatically, peaking at the first bin. (C) The cumulative distribution showed that before applying the toxin the frequency of muscle activity was smaller than $0.5 \mathrm{~Hz}$ in only $15 \%$ of cultures. This fraction increased to approximately $60 \%$ after adding botulinum toxin $A$ to the culture medium. Arrows indicate the medians before (control: $1.40 \mathrm{~Hz}$ ) and after adding incobotulinumtoxin A (toxin: $0.14 \mathrm{~Hz}$ ). 


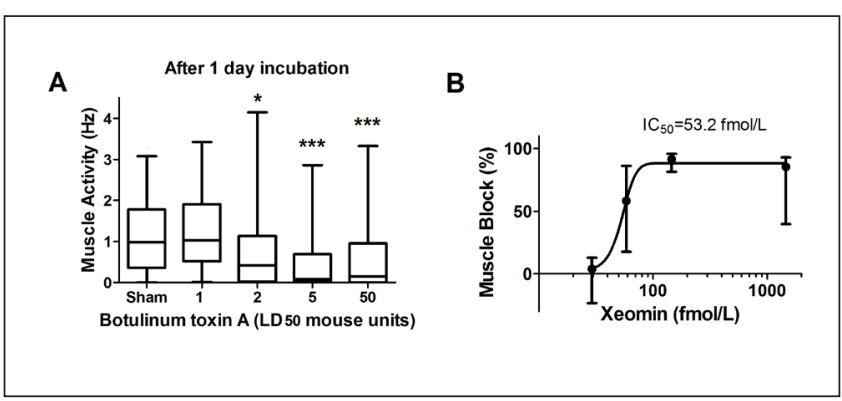

Fig. 4: Muscle activity in botulinum versus sham-treated cultures

(A) The box plots compare spontaneous muscle activity as monitored in cell cultures before and after exposure to $1(n=33)$, $2(n=31), 5(n=45)$ and $50(n=49) L_{50}$ mouse units botulinum toxin and sham treatment $(n=97)$. The medians are represented by the horizontal lines within the boxes. The boxes correspond to the interquartile range and the whiskers to the 2.5-97.5 percentile. For statistical testing of non-parametric data, the Kruskal-Wallis test combined with Dunn's multiple comparison analysis was applied. The frequency of muscle contractions was significantly lower in the botulinum toxin group at 2, 5 and 50 LD $_{50}$ mouse units compared to the sham group ( $\mathrm{p}$-values are indicated on top of the box plots). (B) Concentration-response curve indicating an inhibitory concentration of $50 \%\left(\mathrm{IC}_{50}\right)$ at $53.2 \mathrm{fmol} / \mathrm{l}$. The $95 \%$ confidence intervals of the medians are displayed as bars.

was not different between the botulinum toxin and sham group (Kruskal-Wallis test for unpaired samples). In contrast, $24 \mathrm{~h}$ after adding botulinum toxin $\mathrm{A}$, muscle activity was significantly reduced at concentrations of 2, 5, and $50 \mathrm{LD}_{50}$ mouse units, but remained almost unchanged at 1 LD50 mouse unit (Fig. 4A). Derived from this data set, a concentration-response curve indicated an inhibitory concentration of $50 \%\left(\mathrm{IC}_{50}\right)$ at $53.2 \mathrm{fmol} / 1$ Xeomin $^{\circledR}$ (Fig. 4B).

\subsection{Long-term monitoring of botulinum toxin effects}

In a next step, the muscle-relaxing action of botulinum toxin $\mathrm{A}$ was monitored for up to 3 days. Concentration-dependent effects were very similar after 24 and $72 \mathrm{~h}$ (Fig. 4A, 5A). Interestingly, effects partially only reached significance level after $48 \mathrm{~h}$ (Fig. 5B). Thus, we explored the stability of muscle activity in individual cell cultures on a daily basis. We plotted the frequency of muscle contractions of cultures undergoing sham treatment as determined shortly after exchanging the culture medium and $24 \mathrm{~h}$ later (Fig. 6D). In the middle and on the right hand side similar plots are provided for the subsequent days (Fig. 6E,F). Assuming that muscle activity in individual cell cultures remains stable for $24 \mathrm{~h}$, all points would line up on the diagonal band. However, this could clearly not be observed, indicating that spontaneous muscle activity in individual cultures seems variable. In the upper row of Figure 6 similar plots are provided for the botulinum toxin group. In Figure 6A, a number of points are located on a vertical line on the left hand side of the plots. Muscle activity in these cultures completely
A

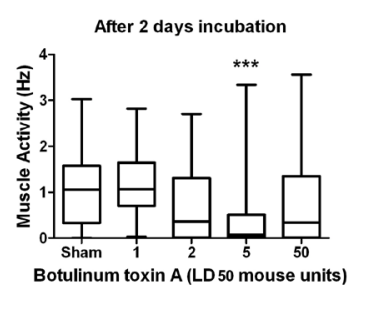

B

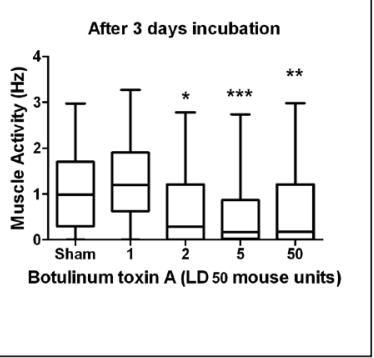

Fig. 5: Botulinum toxin effect after 48 and $72 \mathrm{~h}$

(A) Muscle activity for $1(n=33), 2(n=31), 5(n=45)$, and $50(n=49)$ LD50 mouse units botulinum toxin and sham treatment $(n=97)$ after $48 \mathrm{~h}$ neurotoxin incubation. Note the reduction of frequency at 2, 5, and $50 \mathrm{LD}_{50}$ mouse units, while statistical significance was solely reached at $5 \mathrm{LD}_{50}$ mouse units. (B) After a $72 \mathrm{~h}$ incubation, the botulinum toxin effect was significant at 2, 5, and 50 LD $_{50}$ mouse units.

disappeared $24 \mathrm{~h}$ after treatment with incobotulinumtoxin A. In Figure $6 \mathrm{~B}$ a considerable number of cultures are on a horizontal line on the bottom of the figure. Interestingly, these cultures were inactive $24 \mathrm{~h}$ after adding incobotulinumtoxin A, but regained activity after $48 \mathrm{~h}$. This phenomenon was not observed in the sham group. Homeostatic mechanisms, e.g., an increased expression of nicotinic acetylcholine receptors and other ion channels, may partly account for the spontaneous re-occurrence of muscle activity in these samples (Galante et al., 2000).

In summary, the maximum effect of the neurotoxin was already achieved within the first $24 \mathrm{~h}$ after exposing the cocultures to botulinum toxin A (Fig. 4). Furthermore, spontaneous muscle activity in individual cultures shows large fluctuations when evaluated on a day-to-day basis (Fig. 6), thus introducing considerable scatter into the data set.

\section{Discussion}

\subsection{Salient features of nerve-muscle cultures}

In the present study, nerve-muscle cultures composed of spinal cord and muscle tissue were used for detecting the musclerelaxing action of botulinum toxin $\mathrm{A}$. In these cell cultures, the muscle-relaxant properties of botulinum toxin $\mathrm{A}$ can be assessed by comparing the frequency of muscle contractions in treated versus untreated samples. Our key finding is that at therapeutically relevant concentrations, incobotulinumtoxin A significantly decreased muscle activity (Fig. 3,4). At a concentration of $58 \mathrm{fmol} / 1$ or higher, incobotulinumtoxin A reduced the frequency of muscle contractions within $24 \mathrm{~h}$ after starting the treatment, indicating that nerve-muscle cultures are as sensitive as the mouse LD $_{50}$ assay. Furthermore, the limit of detection, as observed in our study, is close to the most sensitive cell-based bioassays, capable of detecting concentrations of botulinum neurotoxin A ranging between 30 and $50 \mathrm{fmol} / 1$ (Pellett et al., 2007). 


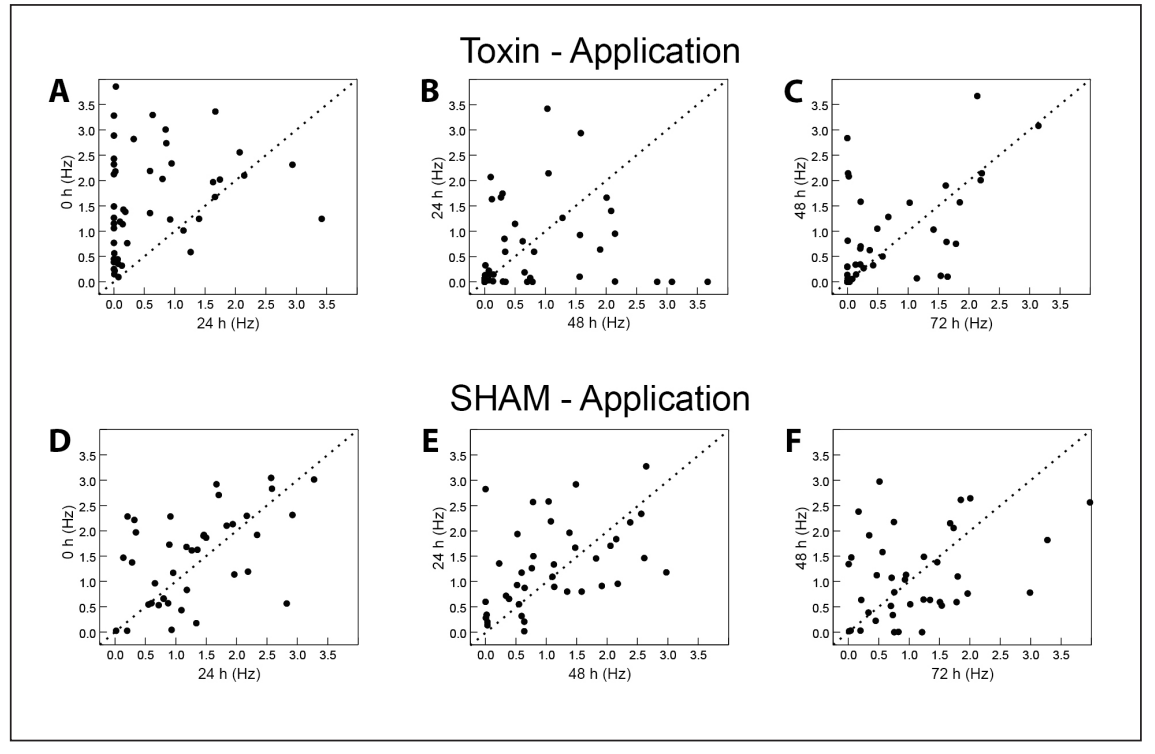

Fig. 6: Muscle activity in individual cultures compared at $\mathbf{2 4} \mathrm{h}$ time lags The frequency of muscle contractions determined in individual cultures at 24 $h$ intervals $(A-F)$. The value of $0 \mathrm{~h}$ that labels the vertical axis of the plots on the left-hand side corresponds to the activity derived from the pre-treatment condition $(A, D)$. Data points located above the dotted diagonal lines indicate that the activity of these cultures decreased within the past $24 \mathrm{~h}$ of the $\mathrm{x}$-scale labeling. The opposite is true for those data points located below the broken lines. Note that as early as $24 \mathrm{~h}$ after botulinum toxin $\mathrm{A}$ treatment the vast majority of cell cultures show a decrease in muscle activity (A).
Nerve-muscle cultures offer a micro-environment displaying all physiological requirements for botulinum toxin to be effective. This includes the binding of the heavy chain to the cell surface receptor, endocytosis, channel formation, conductance of the light chain into the cytosol, refolding of the light chain, proteolytic cleavage of the target protein SNAP-25 and the failure of neuromuscular transmission. Each of these substeps may influence the overall efficacy of botulinum toxins (Schiavo et al., 2000). Therefore, the capability of our cellular assay to monitor holotoxin activity is clearly an advantage over other assays measuring only one property of botulinum neurotoxin A toxicity, such as the cleavage of SNAP-25.

The results of the present study are in accordance with previous reports that primary cultures of neurons isolated either from the spinal cord or from the dorsal root ganglia are quite sensitive to botulinum neurotoxins (Keller et al., 1999; Pellett et al., 2007; Sheridan et al., 2005; Welch et al., 2000). In these investigations the potency of botulinum toxin A was quantified by Western blot analysis of the cleaved target protein SNAP-25 or by probing the amount of neurotransmitter that is released in response to a membrane depolarization caused by raising the extracellular concentration of potassium ions. However, in the spinal cord, different neurons synthesize and release various neurotransmitters such as GABA, glycine, glutamate and acetylcholine. The study of Bigalke and coworkers showed that different spinal neurons are not equally sensitive to botulinum toxins. This raises the question, how these dissimilar actions on GABAergic, glycinergic, glutamatergic and cholinergic synapses can be explained on a molecular level (Bigalke et al., 1985). This question clearly remains to be addressed in further investigations. As muscle activity in nerve-muscle cultures depends on neuromuscular transmission, it is quantitatively linked to the amount of acetylcholine released by spinal motor neurons onto muscle cells. Thus it is likely that the decrease in muscle activity induced by botulinum toxin A as observed in the present study predominantly reflects a diminished release of the neurotransmitter at cholinergic synapses.

Another distinctive feature of organotypic nerve-muscle cultures as compared to other cell culture approaches may result from the involvement of intact neuromuscular synapses that are subject to the actions of botulinum toxins. From studies on muscle-relaxant agents it is well known that blockade of at least $75 \%$ of postsynaptic nicotinic acetylcholine receptors is required to produce signs of muscle relaxation in humans and animals (Jonsson et al., 2006; Tuba et al., 2002). This finding suggests that incomplete cleavage of SNAP-25, e.g., by low concentrations of botulinum toxin $\mathrm{A}$, is detected by Western blot analysis, but may not translate into a decrease in muscle tone. To date, we do not know how the cleavage of the target protein and the dysfunction of the neuromuscular synapse relate to each other in quantitative terms. Thus, assays containing intact neuromuscular synapses, such as the nervus phrenicus diaphragm preparation and nerve-muscle cultures provide a more relevant outcome than assays that simply measure cleavage of SNAP-25, as only the former methods monitor the patient-relevant endpoint, i.e., muscle relaxation.

\subsection{Actions of botulinum toxins at the neuromuscular junction}

Depending on the medical condition, the recommended dosage of incobotulinumtoxin A ranges between 12.5 and 200 mouse $\mathrm{LD}_{50}$ units. We observed that therapeutically relevant concentrations of the neurotoxin caused a significant reduction of muscle activity in nerve-muscle cultures within $24 \mathrm{~h}$ (Fig. 4). This effect persisted for at least $72 \mathrm{~h}$. Interestingly, incobotulinumtoxin A strongly reduced the frequency of muscle contractions but without causing complete failure of muscle activity. Is the latter observation consistent with the molecular actions of botulinum toxin A? In an elegant study Capogna and coworkers analyzed the actions of several botulinum neurotoxins on neurotransmitter release (Capogna et al., 1997). In this investigation, hippo- 
campal tissue cultures were incubated with a high dose of botulinum neurotoxin A of 50-100 ng/ml for more than two days in order to achieve complete cleavage of SNAP-25. As expected, action potential dependent and independent neurotransmitter release was greatly reduced in slices exposed to botulinum toxin A. However, synaptic transmission was partly rescued by increasing the extracellular concentration of $\mathrm{Ca}^{2+}$-ions. Moreover, transmitter release was observed in toxin-treated cultures during repetitive firing of presynaptic neurons, a condition of neuronal activity associated with a high concentration of $\mathrm{Ca}^{2+}$-ions in presynaptic terminals. The conclusion that blockade of synaptic transmission by botulinum toxin $\mathrm{A}$ is overridden by high $\mathrm{Ca}^{2+}$ is also backed by in vivo studies and by investigations using the nervus phrenicus diaphragm preparation (Burgen et al., 1949; Cull-Candy et al., 1976; Humeau et al., 2000). In summary, the results of the present study, namely that incobotulinumtoxin $\mathrm{A}$ reduced muscle activity to a large extent, but did not completely abolish muscle contractions, is clearly in accordance with the reported effects of botulinum toxin A as observed in several experimental preparations.

\subsection{Current limitations of nerve-muscle cultures in neurotoxin potency testing}

A major limitation of nerve-muscle cultures to be used in neurotoxin potency testing is the high degree of variability apparent in our dataset. There are multiple possible sources that may account for the variability in our read-out parameter, exemplified in Figure 6. First, muscle activity is predominantly triggered by ongoing spontaneous action potential firing of spinal motor neurons. However, the number of action potentials generated by these neurons per second may vary between a few and hundreds of events. Thus, recording activity for a short period of time (a few min), as performed in our study, does not provide a good estimation of the average activity over much longer intervals (several h), especially when gaps between subsequent recordings are as large as $24 \mathrm{~h}$. On the other hand, longer recording periods than used in this study would decrease the number of cultures that can be analyzed per day. Another source of variability is provided by actions of botulinum toxin at synapses between spinal neurons. In the present work we eliminated glutamatergic synaptic transmission by applying specific blockers. However, actions of botulinum toxin at GABAergic or glycinergic synapses between spinal neurons are still likely to happen and will alter the firing rates of motor neurons. And, finally, it seems well possible that a small group of muscle fibers autonomously generates muscle contractions in the absence of presynaptic signaling. This population is expected to be resistant to botulinum neurotoxins and thus will increase variability in our datasets.

The use of stimulus-induced instead of spontaneous muscle contractions provides a possible solution to overcome these problems. In a stimulus-based bioassay, muscle activity is evoked by forcing motor neurons to generate action potentials, either by applying short electric current or voltage pulses or by using optogenetic methods. Although technically challenging, this option is promising because it opens ways to eliminate data variability introduced by all sources discussed above.

\section{References}

Adler, S., Bicker, G., Bigalke, H. et al. (2010). The current scientific and legal status of alternative methods to the LD50 test for botulinum neurotoxin potency testing - the report and recommendations of a ZEBET expert meeting. Altern Lab Anim 38, 315-330.

Antonucci, F., Rossi, C., Gianfranceschi, L. et al. (2008). Longdistance retrograde effects of botulinum neurotoxin A. J Neurosci 28, 3689-3696. http://dx.doi.org/10.1523/JNEUROSCI. 0375-08.2008

Avossa, D., Rosato-Siri, M. D., Mazzarol, F. and Ballerini, L. (2003). Spinal circuits formation: a study of developmentally regulated markers in organotypic cultures of embryonic mouse spinal cord. Neuroscience 122, 391-405. http://dx.doi. org/10.1016/j.neuroscience.2003.07.006

Bigalke, H., Dreyer, F. and Bergey, G. (1985). Botulinum a neurotoxin inhibits non-cholinergic synaptic transmission in mouse spinal cord neurons in culture. Brain Res 360, 318324. http://dx.doi.org/10.1016/0006-8993(85)91248-X

Bitz, S. (2010). The botulinum neurotoxin LD50 test - problems and solutions. ALTEX 27, 114-116. http://www.altex. $\mathrm{ch} /$ All-issues/Issue. $50 . \mathrm{html}$ ?iid=113\&aid=4

Braschler, U. F., Iannone, A., Spenger, C. et al.(1989). Amodified roller tube technique for organotypic cocultures of embryonic rat spinal cord, sensory ganglia and skeletal muscle. $\mathrm{J} \mathrm{Neu-}$ rosci Methods 29, 121-129. http://dx.doi.org/10.1016/01650270(89)90023-X

Burgen, A. S. V., Dickens, F. and Zatman, L. J. (1949). The action of botulinum toxin on the neuro-muscular junction. $J$ Physiol 109, 10-24.

Capogna, M., McKinney, R., O'Connor, V. et al. (1997). $\mathrm{Ca}^{2+}$ or $\mathrm{Sr}^{2+}$ partially rescues synaptic transmission in hippocampal cultures treated with botulinum toxin $\mathrm{A}$ and $\mathrm{C}$, but not tetanus toxin. J Neurosci 17, 7190-7202.

Cull-Candy, S. G., Lundh, H. and Thesleff, S. (1976). Effects of botulinum toxin on neuromuscular transmission in the rat. $J$ Physiol 260, 177-203.

Davis, G. (2006). Homeostatic control of neural activity: from phenomenology to molecular design. Annu Rev Neurosci 29, 307-323. http://dx.doi.org/10.1146/annurev. neuro.28.061604.135751

Dressler, D. (2012). Five-year experience with incobotulinumtoxinA (Xeomin ${ }^{\circledR}$ ): the first botulinum toxin drug free of complexing proteins. Eur J Neurol 19, 385-389. http://dx.doi. org/10.1111/j.1468-1331.2011.03559.x

Drexler, B., Seeger, T., Grasshoff, C. et al. (2011). Long-term evaluation of organophosphate toxicity and antidotal therapy in co-cultures of spinal cord and muscle tissue. Toxicol Lett 206, 89-93. http://dx.doi.org/10.1016/j.toxlet.2011.04.010

Drexler, B., Thiermann, H., Antkowiak, B. and Grasshoff, C. (2013). Effects of succinylcholine in an organotypic spinal cordskeletal muscle coculture of embryonic mice. Chem Biol Interact 206, 555-560. http://dx.doi.org/10.1016/j.cbi.2013.06.021

Fernández-Salas, E., Wang, J., Molina, Y. et al. (2012). Botulinum neurotoxin serotype A specific cell-based potency assay to replace the mouse bioassay. PLoS One 7, e49516. http:// dx.doi.org/10.1371/journal.pone.0049516 
Gähwiler, B. H. (1981). Organotypic monolayer cultures of nervous tissue. J Neurosci Methods 4, 329-342. http://dx.doi. org/10.1016/0165-0270(81)90003-0

Galante, M., Nistri, A. and Ballerini, L. (2000). Opposite changes in synaptic activity of organotypic rat spinal cord cultures after chronic block of AMPA/kainate or glycine and GABA A receptors. J Physiol 523, 639-651. http://dx.doi. org/10.1111/j.1469-7793.2000.t01-1-00639.x

Grate, J. W., Ozanich Jr., R. M., Warner, M. G. et al. (2010). Advances in assays and analytical approaches for botulinumtoxin detection. TrAC Trends in Analytical Chemistry 29, 1137-1156. http://dx.doi.org/10.1016/j.trac.2010.07.005

Humeau, Y., Doussau, F., Grant, N. J. and Poulain, B. (2000). How botulinum and tetanus neurotoxins block neurotransmitter release. Biochimie 82, 427-446. http://dx.doi.org/10.1016/ S0300-9084(00)00216-9

Jonsson, M., Gurley, D., Dabrowski, M. et al. (2006). Distinct pharmacologic properties of neuromuscular blocking agents on human neuronal nicotinic acetylcholine receptors: a possible explanation for the train-of-four fade. Anesthesiology 105, 521-533. http://dx.doi.org/10.1097/00000542-20060900000016

Keller, J. E., Neale, E. A., Oyler, G. and Adler, M. (1999). Persistence of botulinum neurotoxin action in cultured spinal cord cells. FEBS Lett 456, 137-142. http://dx.doi.org/10.1016/ S0014-5793(99)00948-5

Magloire, V. and Streit, J. (2009). Intrinsic activity and positive feedback in motor circuits in organotypic spinal cord slice cultures. Eur J Neurosci 30, 1487-1497. http://dx.doi. org/10.1111/j.1460-9568.2009.06978.x

Martens, S. and McMahon, H. T. (2008). Mechanisms of membrane fusion: disparate players and common principles. Nat Rev Mol Cell Biol 9, 543-556. http://dx.doi.org/10.1038/ nrm2417

Pellett, S., Tepp, W. H., Clancy, C. M. et al. (2007). A neuronal cell-based botulinum neurotoxin assay for highly sensitive and specific detection of neutralizing serum antibodies. FEBS Letters 581, 4803-4808. http://dx.doi.org/10.1016/j. febslet.2007.08.078

Pharm EU - European Pharmacopoeia (2011) 7.2. Botulinum toxin type A for injection (2011). 3613-3615.

Rosato-Siri, M. D., Zoccolan, D., Furlan, F. and Ballerini, L. (2004). Interneurone bursts are spontaneously associated with muscle contractions only during early phases of mouse spinal network development: a study in organotypic cultures. Eur J Neurosci 20, 2697-2710. http://dx.doi.org/10.1111/j.14609568.2004.03740.x

Scarlatos, A., Cadotte, A. J., DeMarse, T. B. and Welt, B. A. (2008). Cortical networks grown on microelectrode arrays as a biosensor for botulinum toxin. J Food Sci 73, E129-E136. http://dx.doi.org/10.1111/j.1750-3841.2008.00690.x

Schiavo, G., Matteoli, M. and Montecucco, C. (2000). Neurotoxins affecting neuroexocytosis. Physiol Rev 80, 717-766.

Sheridan, R. E., Smith, T. J. and Adler, M. (2005). Primary cell culture for evaluation of botulinum neurotoxin antagonists. Toxicon 45, 377-382. http://dx.doi.org/10.1016/ j.toxicon.2004.11.009
Spenger, C., Braschler, U. F., Streit, J. and Luscher, H. R. (1991). An organotypic spinal cord - dorsal root ganglion skeletal muscle coculture of embryonic rat. I. The morphological correlates of the spinal reflex arc. Eur J Neurosci 3, 1037-1053. http://dx.doi.org/10.1111/j.1460-9568.1991.tb00041.x

Stoppini, L., Buchs, P.-A. and Muller, D. (1991). A simple method for organotypic cultures of nervous tissue. $\mathrm{J} \mathrm{Neu}$ rosci Methods 37, 173-182. http://dx.doi.org/10.1016/01650270(91)90128-M

Streit, J., Spenger, C. and Luscher, H. R. (1991). An organotypic spinal cord - dorsalroot ganglion - skeletal muscle coculture of embryonic rat. II. Functional evidence for the formation of spinal reflex arcs in vitro. Eur J Neurosci 3, 1054-1068. http:// dx.doi.org/10.1111/j.1460-9568.1991.tb00042.x

Talpalar, A. E. and Kiehn, O. (2010). Glutamatergic mechanisms for speed control and network operation in the rodent locomotor CpG. Front Neural Circuits 4, 19. http://dx.doi. org/10.3389/fncir.2010.00019

Tuba, Z., Maho, S. and Vizi, E. (2002). Synthesis and structure-activity relationships of neuromuscular blocking agents. Curr Med Chem 9, 1507-1536. http://dx.doi. org/10.2174/0929867023369466

Turrigiano, G. G. (1999). Homeostatic plasticity in neuronal networks: the more things change, the more they stay the same. Trends Neurosci 22, 221-227. http://dx.doi.org/10.1016/ S0166-2236(98)01341-1

Turton, K., Chaddock, J. A., Acharya, K. R. (2002). Botulinum and tetanus neurotoxins: structure, function and therapeutic utility. Trends Biochem Sci 27, 552-558. http://dx.doi. org/10.1016/S0968-0004(02)02177-1

Welch, M. J., Purkiss, J. R. and Foster, K. A. (2000). Sensitivity of embryonic rat dorsal root ganglia neurons to Clostridium botulinum neurotoxins. Toxicon 38, 245-258. http://dx.doi. org/10.1016/S0041-0101(99)00153-1

\section{Conflict of interest}

The authors declare that no conflict of interest exists.

\section{Acknowledgements}

We thank Claudia Holt for excellent technical assistance. This work was supported by grant BfR FK31328-456 (Federal Institute for Risk Assessment).

\section{Correspondence to}

Veit-Simon Eckle, MD

Department of Anesthesiology and Intensive Care

Experimental Anesthesiology Section

Eberhard-Karls University Tübingen

Waldhörnlestr. 22

72072 Tübingen, Germany

Phone: +4970712981159

Fax: +4970712925073

e-mail: veit-simon.eckle@uni-tuebingen.de 